○

CHOICE

${ }^{1}$ Liverpool Ocular Oncology Centre, St Paul's Eye Clinic, Royal Liverpool University Hospital, Liverpool, UK; ${ }^{2}$ Departments of Pathology/ Ophthalmology, University of Liverpool, Liverpool, UK

Correspondence to:

Professor B Damato, Ocular

Oncology Service, Royal

Liverpool University Hospital,

Prescot Street Liverpool L7 8XP.

UK

bertil@damato.co.uk

Presented at the International Conference on Ocular Oncology, Siena, Italy, June 2007 and at the European Vision and Eye Research (EVER) Association meeting, Portoroz, Slovenia, October 2007

Accepted 31 October 2007 Published Online First 31 July 2008

\title{
Clinical mapping of conjunctival melanomas
}

\author{
B Damato, ${ }^{1}$ S E Coupland ${ }^{2}$
}

\section{ABSTRACT}

Aim: To develop a system for clinically mapping conjunctival melanomas, taking into account the tumour's stage of development, location and extent.

Methods: Baseline clinical features of 40 patients with conjunctival melanoma, first treated at our centre between 1993 and 2006, were studied. Maps showing sectorial location and circumferential spread in all areas of conjunctiva were digitally generated from numerical data. Results: The patients (18 female; 22 male) had a median age of 59 years. Three had multiple tumours and 20 had mixed, invasive and intraepithelial disease. The Tumour Node and Metastasis (TNM) T stage was I in 11 patients, II in 20, III in eight and IV in one. The tumour was medial in 19\% of eyes with T stages I and II as compared with $67 \%$ of eyes with stages III and IV (Mann-Whitney, $<0.001$ ). Within each T stage of the TNM system, there was considerable variation in tumour dimensions, circumferential spread and longitudinal extent.

Conclusions: A system for clinically mapping conjunctival tumour location and extent has been developed. This revealed tumour variation within TNM T stages and may facilitate better collection of data for multicentre trials.

Conjunctival melanomas are rare. ${ }^{1}$ They may be associated with primary acquired melanosis (PAM), which we prefer to call "conjunctival in situ melanoma." Exenteration has been superseded by local excision of any nodules together with adjunctive therapy consisting of cryotherapy, topical chemotherapy and radiotherapy, individually or in

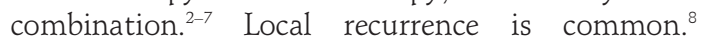
Metastasis can occur to regional nodes and systemically. Sentinel node biopsy is controversial. ${ }^{9}$ The 10 year melanoma-related mortality is $25-30 \%$ and is higher with involvement of non-bulbar conjunctiva, local tumour recurrence, multifocal tumours, increased tumour thickness, high mitotic rate, epithelioid cells and lymphatic invasion. ${ }^{8}{ }^{10-13}$

The sixth version of the Tumour Node and Metastasis (TNM) staging system of the American Joint Committee on Cancer/International Union Against Cancer (AJCC/UICC) categorises conjunctival tumours only according to their degree of invasion and radial extent but has not gained widespread acceptance. ${ }^{14}$

The aim of this study was to develop a system for mapping conjunctival melanomas.

\section{PATIENTS AND METHODS}

Patients were included in this study if treated for conjunctival melanoma between January 1993 and December 2006. They were excluded if they had undergone any surgery before referral or if the charts were no longer available (one patient).

Ocular examination included visual acuity and intraocular pressure measurements, inspecting the external eye, anterior segment and fundus with slit-lamp biomicroscopy and indirect ophthalmoscopy. Each sector of the bulbar and inferior tarsal conjunctiva was examined by retracting the eyelid and asking the patient to look in the opposite direction. The superior tarsal conjunctiva and fornix were examined with a magnifying lens, gently pulling the eyelid away from the eye (hence avoiding double eversion of the eyelid). If a more detailed examination of the superior tarsal conjunctiva was indicated, this was done with a slitlamp after everting the upper lid. Regional lymph nodes were palpated routinely. Patients were photographed routinely. Towards the end of the study, a formal protocol was instituted, imaging each sector of the conjunctiva in turn, with the patient looking away from that sector, retracting the eyelids to expose the fornices, and everting the eyelids to photograph the superior and inferior palpebral conjunctiva. A ruler was held against the lower lid to calibrate photographs.

The patients were identified by searching the computerised database of the Liverpool Ocular Oncology Centre and archives of the pathology department at the Royal Liverpool University Hospital. All case notes, computerised records, histological sections and clinical photographs were reviewed to define tumour location and extent. If not documented adequately in the case notes at the time of initial assessment, measurements such as basal dimensions and distances from limbus and lid margin were made from photographs. Calibration for such measurements was based on corneal diameter, which was assumed to be $12 \mathrm{~mm}$. Tumour thickness and width were determined histologically. ${ }^{15}$ Sections were stained using $\mathrm{H} \& \mathrm{E}$ and immunohistochemically. ${ }^{16}{ }^{17}$

Clinical and histological data were summarised on proformas and computerised in the ocular oncology database, which was customised for conjunctival tumours. The data were then exported to SPSS (Chicago) for analysis. Tumour location and extent were mapped using an Excel spreadsheet (Microsoft Windows, Seattle, WA). This was done by generating a table in SPSS, with a score of 1 or 0 allocated to each clock hour of every region of conjunctiva, according to whether or not this was affected by the tumour in question. This was done for up to three tumours per patient. The tables were transferred from SPSS to Excel so that bubble plots could be generated, with invasive melanoma depicted in grey and pigmented intraepithelial disease with a striped texture. Cumulative bubble plots with data from groups of patients (eg, with or without metastatic death) were generated, with the bubble sizes representing either the number or the proportion of patients in the group having invasive tumour in the relevant conjunctival area. 
The centre of the conjunctiva was defined as the central cornea, with limbus, bulbar conjunctiva, fornix, tarsal conjunctiva, eyelid margin and skin considered as being progressively more peripheral. The plica was regarded as being an extension of forniceal conjunctiva, and the caruncle was considered to be at the same eccentricity as tarsal conjunctiva. Circumferential spread along limbus, bulbar conjunctiva, fornix, tarsal conjunctiva, eyelid margin and adjacent skin was measured in clock minutes (which are different from minutes of arc). Longitudinal and transverse basal tumour dimensions were documented in millimetres. For tumours involving bulbar conjunctiva, distance between central tumour margin and limbus was measured in millimetres, with negative values if the tumour extended into cornea. For tarsal tumours, distance between peripheral tumour margin and inner eyelid margin was measured, with negative values if this margin was located within conjunctiva and positive values if it was in skin.

Our proforma was designed to store details on up to five tumours per patient. The largest tumour was regarded as the primary lesion. Invasive melanoma and intraepithelial disease in the same eye were considered separately, even if confluent. Clinically, only pigmented intraepithelial melanocytic proliferation could be mapped, with such "primary acquired melanosis (PAM)" subsequently categorised histologically as (1) PAM without atypia, (2) PAM with atypia resulting in invasive melanoma or (3) secondary, diffuse, superficial spread from a nodular melanoma. We now refer to "PAM without atypia" and "PAM with atypia" as "conjunctival melanocytic hyperplasia" and "conjunctival in situ melanoma" respectively.

Invasive tumours were defined as being pigmented or amelanotic. Secondary effects included features such as feeder vessels and inflammation.

The Tenets of the Helsinki Declaration were followed. Patient consent was routinely obtained for the use of data, images and tissues for research, audit and teaching. Ethical Committee approval was not required.

\section{RESULTS}

\section{Baseline data}

The cohort comprised 40 patients (18 female; 22 male) with a median age of 59 years (range 24 to 88 years). The tumour involved the right eye in 18 patients and the left eye in 22 . Three patients had multiple tumours, and 20 had mixed tumours (ie, invasive melanoma and intraepithelial disease) (fig 1). The centre of the largest tumour was located in the following sectors: superior (3); supero-temporal (1); temporal (18); inferotemporal (4); inferior (2); infero-nasal (4); nasal (4); and superonasal (4). The median largest basal tumour diameter was $6.8 \mathrm{~mm}$ (range 3 to 25), and the median tumour thickness was $2.2 \mathrm{~mm}$ (range 0.1 to 15.5 ). The central tumour margin was located in cornea (20 patients), limbus (6), bulbar conjunctiva (12), tarsal conjunctiva (1) and caruncle (1). The peripheral tumour margin was located in bulbar conjunctiva (31), plica (1), caruncle (5), tarsal conjunctiva (1), eyelid margin (1) and skin (1). Six tumours involved the caruncle, with five of these also involving the plica. Non-statistically, bubble plots suggested no great interocular or gender differences but indicated that medial conjunctival involvement correlated with local tumour recurrence and metastatic death (fig 2). The TNM stage was: I (11 patients); II (20 patients); III (eight patients); and IV (one patient). Figure 3 shows the basal tumour dimensions, tumour height, circumferential tumour extent and, for bulbar tumours, the tumour-to-limbus distance, according to TNM stage. In stages T1 and T2, the tumour location was medial in six (19\%),

\section{A clinical mapping system for conjunctival melanoma}

- Displays the entire conjunctiva as a flat surface, with the central point located at the centre of the cornea and concentric regions such as limbus, bulbar conjunctiva, fornix, palpebral conjunctiva and eyelid considered progressively more peripheral.

- Plots whether or not each clock hour of every region is affected by invasive melanoma (solid fill) or intraepithelial disease (hatched drawing).

- Maps are drawn digitally from numerical data, allowing cumulative plots comparing groups of patients.

- Clinical mapping improves documentation of tumour size, location and extent, and should therefore facilitate data collection for multicentre trials.

midline in two $(7 \%)$ and temporal in $23(74 \%)$ as compared with medial in six (67\%) and midline in three $(33 \%)$ in stages T3 and T4 (Mann-Whitney $\mathrm{U}=39 ; \mathrm{z}=3.68 ; \mathrm{p}<0.001$ ).

\section{DISCUSSION}

We have developed a system for mapping conjunctival melanomas clinically (table 1). Tumours were drawn from numerical data, which enabled cumulative plots to be generated, thereby allowing different subgroups of patients to be compared visually.

Staging of cancer progression at the time of initial treatment is fundamental to prognostication, treatment selection and clinical trials. The sixth version of the TNM staging system classifies conjunctival melanomas as: TI for tumours involving only the bulbar conjunctiva; TII for tumours with corneal involvement; TIII for tumours involving fornix, caruncle or tarsal conjunctiva; and TIV for tumours invading the eye, orbit, central nervous system or skin. Our study revealed shortcomings of the TNM staging system. First, our cumulative plots (fig 2) showed how local tumour recurrence and metastasis correlated with involvement of medial conjunctiva. Second, as shown in fig 3, our mapping demonstrated that within each $\mathrm{T}$ stage, there was marked variation in tumour dimensions, circumferential spread and longitudinal extent, which are known to have prognostic significance. We have other concerns regarding the TNM staging system. First of all, we are not aware of any evidence indicating that tumours involving cornea have a worse prognosis than those occurring only in bulbar conjunctiva; if anything, the prognosis should be better, because of the absence of lymphatics. ${ }^{18}$ Second, we intuitively suspect that tumours invading the central nervous system have a worse prognosis from those involving only the eyelid skin. For all these reasons, we suspect that important prognostic information is not fully utilised by the TNM system. The TNM system has recently been revised, and the next edition ${ }^{19}$ will take account of our findings. Large, multicentre studies are needed to determine how circumferential tumour location and spread correlate with survival and how continuous variables should be categorised. Such studies will require accurate documentation of tumour location and extent, which we hope will be facilitated by our proposed mapping system.

Several authors have shown a correlation between nonepibulbar tumour location and metastatic disease, but without measuring circumferential spread. ${ }^{811}{ }^{12}$ In their evaluation of risk factors for metastatic death and other outcomes, Shields et 


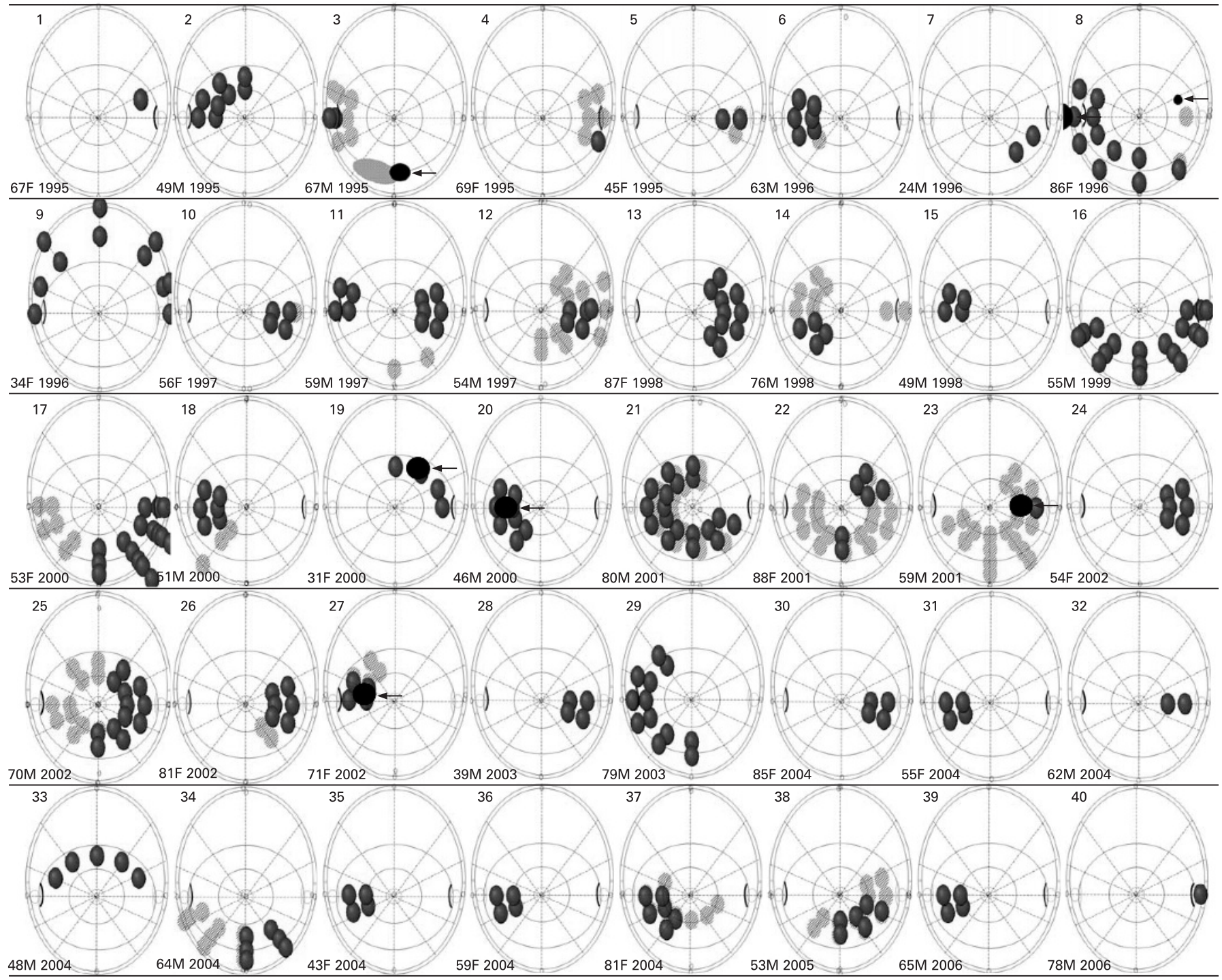

Figure 1 Maps of 40 conjunctival melanomas, showing computer-generated plots of tumour location and extent with invasive melanoma in dark grey and intraepithelial disease in light grey, and recurrent disease (manually drawn) in black. Each spot represents involvement of a sector of conjunctiva and not a separate tumour.

al look at circumferential spread, but only in bulbar conjunctiva and not in fornix and tarsal conjunctiva, which seem more relevant to survival probability. ${ }^{13}$ We measured circumferential spread in all conjunctival regions: limbus, bulbar conjunctiva, palpebral conjunctiva, eyelid margin and skin. Such data are likely to be useful not only in predicting survival but also in evaluating ocular results following treatment of the conjunctival tumour. We preferred clock minutes to clock hours, because clock minutes may be more precise, also avoiding the need for fractions or decimal points.

The number of patients in our study is small compared with other investigations. This is because, unlike other studies, we excluded all patients who had undergone any conjunctival tumour surgery elsewhere before referral to our centre. There are several reasons for this. First, in many such patients, we were unable to define the presenting tumour, because it had already been excised. Second, patients referred with recurrent tumours may have had more aggressive disease so that they may have introduced selection bias into our results. Third, outcomes may have been worsened by suboptimal surgery elsewhere, as previously suggested..$^{20}$
A shortcoming of our study is that the tumour features at initial presentation were defined retrospectively, and this was because we conceived our mapping system only recently. Nevertheless, with the exception of the tumour-to-lid-margin, we felt we were able to describe most tumours adequately from the patient records and photographs. Another limitation is that the patients referred to us with untreated conjunctival melanoma may not have been representative of all individuals developing this disease. Prospective, multicentre studies are needed, with patients being examined and photographed according to a standardised protocol. Such a protocol would also need to specify how primary acquired melanosis should be defined and graded, and how histological data should be added to any clinical maps.

The computer-generated maps shown in fig 1 showed only whether a clock hour of a particular conjunctival region was involved by tumour. Such a low resolution did not allow a full description of tumour shape and did not define the precise location of the central, peripheral and lateral tumour margins. Furthermore, it did not identify two distinct tumours if these were located close together. More detailed mapping using our 
Figure 2 Tumour location and extent according to (A) laterality; (B) sex; (C) local tumour recurrence; and (D) metastatic death. In (B), (C) and (D), tumours in the left eye are transposed to the right eye. In $(A)$ and $(B)$, the bubble size represents the number of cases, and in (C) and (D), the bubble size represents the proportion of cases.

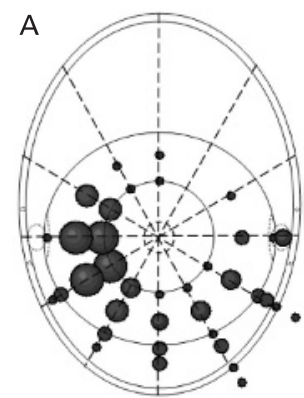

Right eye (18)

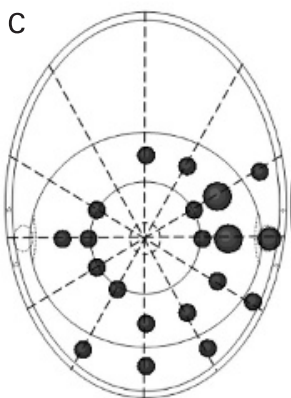

Recurrence (6)

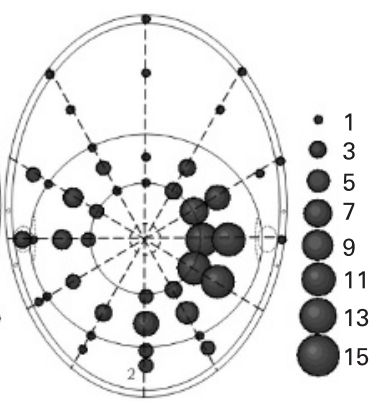

Left eye (22)

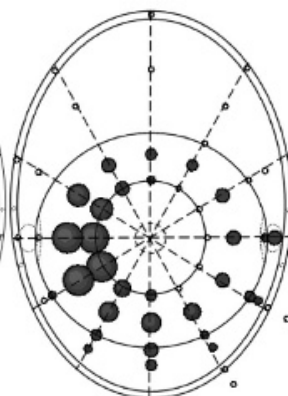

No recurrence (34)

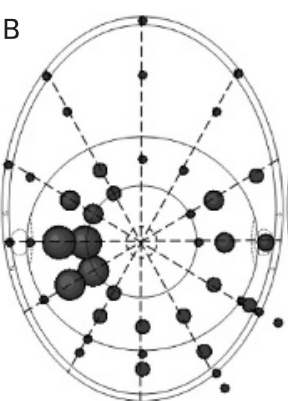

Female (18)

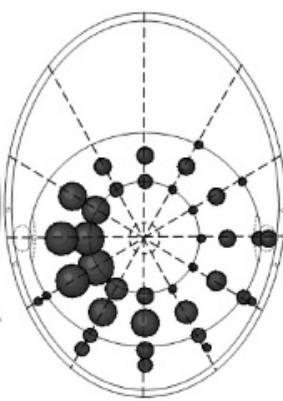

Male (22)

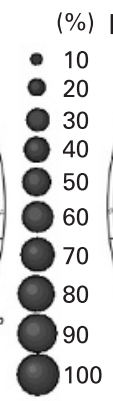

$(\%) \mathrm{D}$
10
20
30
40
50
60
70
80
90
100

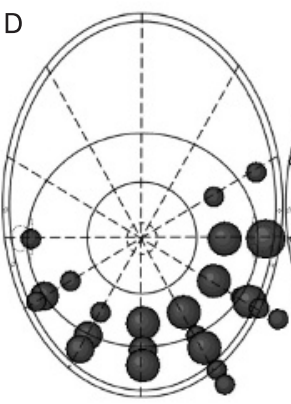

Met death (4)

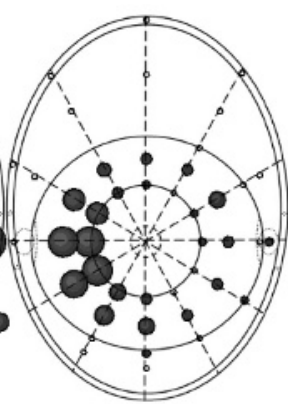

No met death (36) current methods would be feasible but may prove excessively laborious. We are hoping to develop a more user-friendly program for mapping conjunctival tumours more precisely. This will hopefully take into account not only the curvature of the eye but also the maximal radial conjunctival dimensions that occur with eccentric gaze. We measured tumour thickness from histological sections, which is not ideal because of a slight reduction in tissue volume after fixation and because of the risk of tangential cuts. However, in the vast majority of cases, orthograde sections were achieved, providing accurate measurements. In the case of conjunctval melanomas associated with surrounding inflammation, immunohistochemical staining
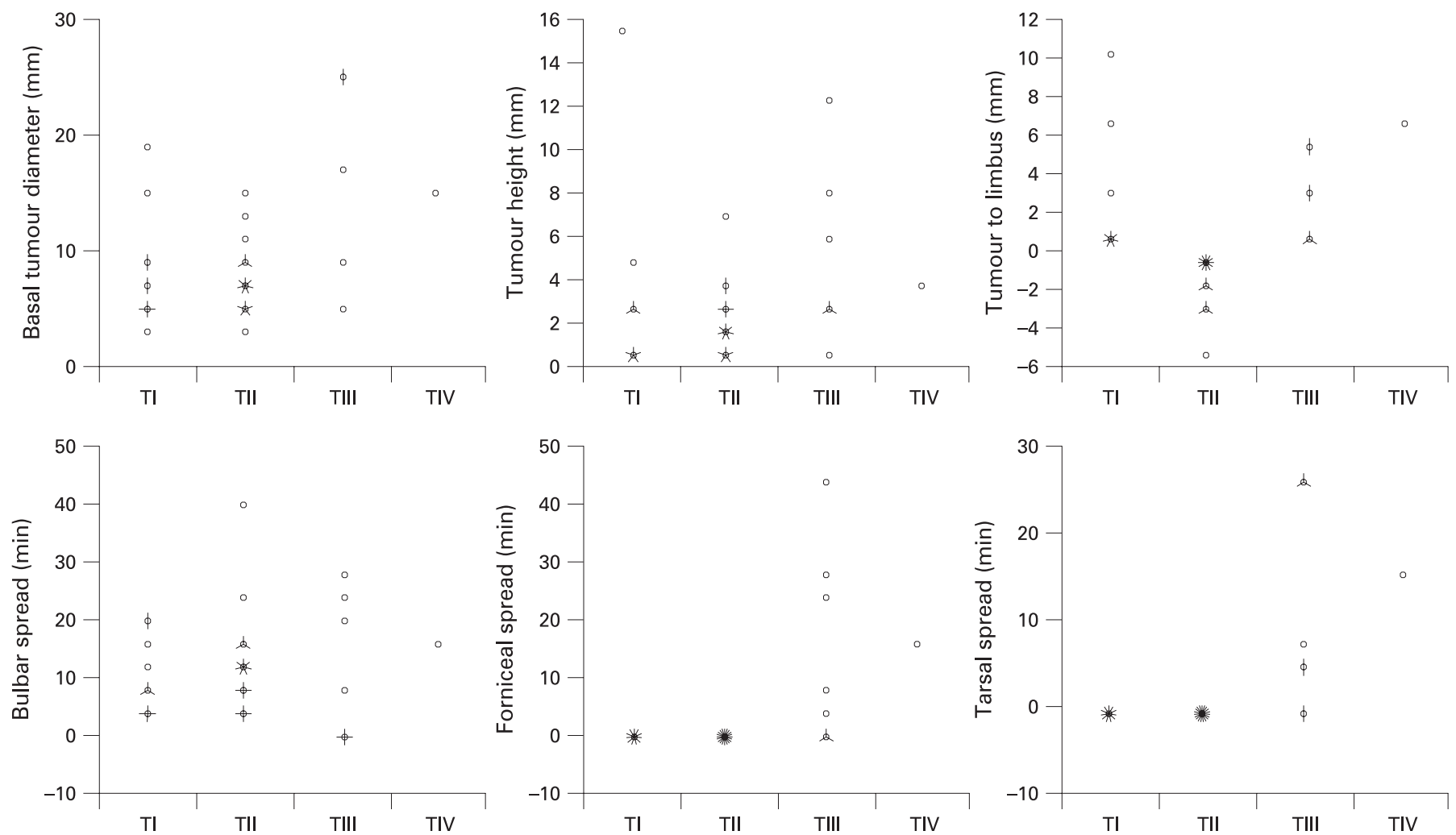

Figure 3 Basal tumour diameter and tumour height, tumour-to-limbus distance, and circumferential tumour extent according to the Tumour Node and Metastasis T stage. Only one patient (case 17) had a TIV tumour (see fig 2). Tumours in the left eye were transposed to the right eye. 
highlighted the tumour cell population, enabling better identification of the tumour dimensions. A method using highfrequency ultrasonography has been described. ${ }^{21}$ We have recently used this technique to measure bulbar tumours, but further evaluation is needed, especially with non-bulbar tumours.

We have not reported outcomes, which were not relevant to the description of our mapping system. These are published elsewhere. ${ }^{22}$ The cumulative plots in fig 2 were shown only to demonstrate the potential of our mapping system. Our treatment outcomes, including local tumour recurrence and metastatic death, have been reported elsewhere.

In conclusion, mapping may facilitate data collection for multicentre trials. There is scope for evaluating and refining this system, not only with melanomas but also with other conjunctival cancers, ideally in several different centres.

Funding: The Ocular Oncology Service is funded by the National Commissioning Group of the National Health Service, which had no role in the design or conduct of the research.

Competing interests: None.

Patient consent: Obtained.

\section{REFERENCES}

1. Seregard S. Conjunctival melanoma. Surv Ophthalmol 1998;42:321-50.

2. Shields JA, Shields CL, De Potter P. Surgical management of conjunctival tumors. The 1994 Lynn B. McMahan Lecture. Arch Ophthalmol 1997;115:808-15.

3. Stannard CE, Sealy GR, Hering ER, et al. Malignant melanoma of the eyelid and palpebral conjunctiva treated with iodine-125 brachytherapy. Ophthalmology 2000;107:951-8

4. Demirci H, McCormick SA, Finger PT. Topical mitomycin chemotherapy for conjunctival malignant melanoma and primary acquired melanosis with atypia: clinical experience with histopathologic observations. Arch Ophthalmol 2000;118:885-91.

5. Finger PT. "Finger-tip" cryotherapy probes: treatment of squamous and melanocytic conjunctival neoplasia. Br J Ophthalmol 2005;89:942-5.
6. Wuestemeyer H, Sauerwein W, Meller D, et al. Proton radiotherapy as an alternative to exenteration in the management of extended conjunctival melanoma. Graefes Arch Clin Exp Ophthalmol 2006;244:438-46.

7. Frucht-Pery J, Pe'er J. Use of Mitomycin $C$ in the treatment of conjunctival primary acquired melanosis with atypia. Arch Ophthalmol 1996;114:1261-4.

8. Missotten GS, Keijser S, de Keizer RJ, et al. Conjunctival melanoma in the Netherlands: a nationwide study. Invest Ophthalmol Vis Sci 2005:46:75-82.

9. Amato M, Esmaeli B, Ahmadi MA, et al. Feasibility of preoperative lymphoscintigraphy for identification of sentinel lymph nodes in patients with conjunctival and periocular skin malignancies. Ophthal Plast Reconstr Surg 2003;19:102-6.

10. Desjardins L, Poncet $P$, Levy $C$, et al. Prognostic factors in malignant melanoma of the conjunctiva. An anatomo-clinical study of 56 patients. J Fr Ophtalmol 1999;22:315-21.

11. Anastassiou G, Heiligenhaus A, Bechrakis N, et al. Prognostic value of clinical and histopathological parameters in conjunctival melanomas: a retrospective study. Br J Ophthalmol 2002;86:163-7.

12. Werschnik C, Lommatzsch PK. Long-term follow-up of patients with conjunctival melanoma. Am J Clin Oncol 2002;25:248-55.

13. Shields CL, Shields JA, Gunduz K, et al. Conjunctival melanoma: risk factors for recurrence, exenteration, metastasis, and death in 150 consecutive patients. Arch Ophthalmol 2000;118:1497-507.

14. Sobin L, Wittekind C. TNM classification of malignant tumours. 6th edn. New York: Wiley-Liss, 2002

15. Tuomaala S, Eskelin S, Tarkkanen A, et al. Population-based assessment of clinical characteristics predicting outcome of conjunctival melanoma in whites. Invest Ophthalmol Vis Sci 2002;43:3399-408.

16. Keijser S, Missotten GS, Bonfrer JM, et al. Immunophenotypic markers to differentiate between benign and malignant melanocytic lesions. $\mathrm{Br} \mathrm{J}$ Ophthalmol 2006;90:213-17.

17. Glasgow BJ, McCall LC, Foos RY. HMB-45 antibody reactivity in pigmented lesions of the conjunctiva. Am J Ophthalmol 1990;109:696-700.

18. Tuomaala $\mathbf{S}$, Aine E, Saari KM, et al. Corneally displaced malignant conjunctival melanomas. Ophthalmology 2002;109:914-19.

19. Seregard S, McGovern H, Coupland SE, et al. TNM staging of conjunctival melanoma. In: Sobin LH, Wittekind Ch, eds. TNM classification of malignant tumours. 7th edn. New York: Wiley Liss. In Press.

20. Shields CL. Conjunctival melanoma. Br J Ophthalmol 2002;86:127.

21. Ho VH, Prager TC, Diwan $\mathrm{H}$, et al. Ultrasound biomicroscopy for estimation of tumor thickness for conjunctival melanoma. J Clin Ultrasound 2007;35:533-7.

22. Damato B, Coupland SE. An audit of conjunctival melanoma treatment in Liverpool. Eye. Published Online First: 6 June 2008. doi:10.1038/eye.2008.154. 